\title{
Contract and move
}

DOI:

$10.1038 /$ nrc2032

\section{URLs \\ Rho}

http://www.ncbi.nlm.nih.gov/ entrez/query.fcgi?db=gene\&c $\mathrm{md}=$ RetrieveEdopt=full_ report\&list_uids $=29984$

ROCK

http://www.ncbi.nlm.nih.gov/ entrez/query.fcgi?db=gene\&c $\mathrm{md}=$ Retrieve\&dopt=full report\&list_uids $=6093$

MLC2

http://www.ncbi.nlm.nih.gov/ entrez/query.fcgi? $\mathrm{CMD}=$ search\&DB=gene

\section{ENDO180}

http://www.ncbi.nlm.nih.gov/ entrez/query.fcgi?db=gene\&c $\mathrm{md}=$ RetrieveEdopt=full_ report\&list_uids $=9902$
The regulated assembly and disassembly of cell-extracellular matrix junctions (focal adhesions) contributes to cell motility and tumour invasion. Rho-ROCK signalling promotes focal adhesion disassembly at the rear of the cell by inducing contractile forces that are dependent on the phosphorylation of myosin light chain 2 (MLC2). Isacke and colleagues now show that the Rho-ROCK-MLC2 signalling pathway is activated to generate contractile forces at distinct subcellular locations through endosomal trafficking.

Using human cancer cell lines the authors compared the small interfering RNA (siRNA)-mediated depletion of the promigratory transmembrane receptor ENDO180 (also known as CD280) and two other endocytic receptors. ENDO180 is constitutively recruited into clathrin-coated pits on the cell surface, which are then internalized into intracellular endosomes.

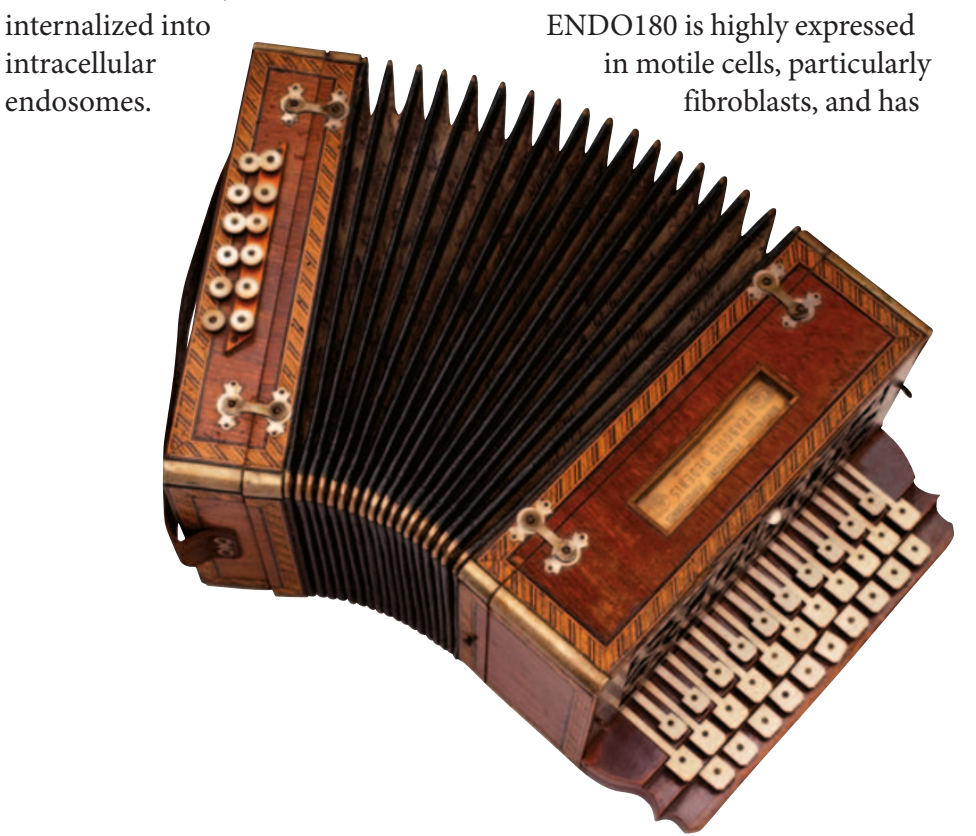

Only the depletion of ENDO180 induced tail-retraction defects, indicative of reduced Rho-ROCKMLC2-mediated contractile forces. This phenotype was comparable to that observed when cells were treated with Rho or ROCK inhibitors, establishing ENDO180-RhoROCK-MLC2 as a functional pathway. Moreover, ENDO180 siRNA or a ROCK inhibitor reduced the phosphorylation of three other ROCK substrates, LIM kinases 1 and 2 , and myosin phosphatase 1 .

Isacke and colleagues also show that ENDO180 is required for the spatial activation of Rho-ROCKMLC2. Using cell lines that stably express an ENDO180 mutant that cannot be internalized, the authors show that ROCK cannot be recruited to focal adhesions. This shows that intracellular ENDO180-containing endosomes are required to activate Rho-ROCK.

ENDO180 is highly expressed an established role in collagen internalization. Thomas Bugge and colleagues have shown that mice undergoing polyomavirus-induced mammary carcinogenesis that also have a targeted deletion in ENDO180 have impaired tumour expansion, and therefore a reduced tumour burden. Isacke and colleagues suggest that this reduction in tumour burden could arise both from the inability of ENDO180-null fibroblasts to remodel the extracellular matrix and from impaired motility within the tumour. Indeed, targeting ENDO180 might benefit anticancer therapy by causing the retention of a tumourinhibitory matrix.

Gemma Alderton

ORIGINAL RESEARCH PAPER Sturge, J., Wienke, D. \& Isacke, C. M. Endosomes generate localized Rho-ROCK-MLC2-based contractile signals via Endo 180 to promote adhesion disassembly.J. Cell Biol. 175, 337-347 (2006) FURTHER READING Curino, A. C. et al. Intracellular collagen degradation mediated by UPARAP/Endo 180 is a major pathway of extracellular matrix turnover during malignancy. J. Cell Biol. 169, 977-985 (2005) 